\title{
Osteopathic Manipulative Treatment for Pediatric Patients With Otitis Media
}

Kat Winger, OMS III; Tami Hendriksz, DO; Kimberly Wolf, DO; Joel Talsma, MS; Stacey Pierce-Talsma, DO

From Touro University College of Osteopathic Medicine-CA in Vallejo, California.

Financial Disclosures:

None reported.

Support: This video was produced by Touro University

College of Osteopathic

Medicine-CA.

Address correspondence to

Tami Hendriksz, DO, 1310

Club Dr, Mare Island, Vallejo,

CA 94592-1187.

Email: tami.hendriksz@tu.edu

Submitted

November 6, 2019;

revision received

January 2, 2020;

accepted

January 27, 2020.
$\mathrm{T}$ he use of osteopathic manipulative treatment (OMT) in children presents an opportunity for a holistic, multidisciplinary approach in the management of pediatric conditions. Pediatric patients are a unique population, as their maturing anatomy differs from that of adults. Osteopathic manipulative treatment is safely used in pediatric patients from infants to adolescents for a wide variety of diagnoses covering common pediatric conditions, including nonmusculoskeletal diagnoses, such as asthma, behavioral problems, and otitis media. ${ }^{1-2}$

Otitis media is the most common reason for children ages 5 years and younger to visit the doctor, and by age 2 years, $90 \%$ of children will have experienced an episode of acute otitis media $(\mathrm{AOM}) .^{3-4}$ Studies have shown that adjunctive OMT can help decrease the duration of AOM and its risk for recurrence. ${ }^{6}$ A pilot study with 1 year of follow-up showed that approximately $62 \%$ of pediatric patients who received 3 weekly OMT sessions after an episode of AOM did not have recurrence of AOM symptoms, whereas other studies found quicker resolution of middle ear effusion using standard care and OMT vs standard care alone. $^{4-6}$

Eustachian tube dysfunction is a known contributing factor for AOM. The eustachian tube connects the middle ear to the nasopharynx and allows for pressure equilibration between the ear and the external environment via the process of yawning, sucking, or swallowing. The eustachian tube begins in the nasopharynx where it contacts 4 muscles that assist in its functionality: tensor veli palatini, medial pterygoid, levator veli palatini, and the salpingopharyngeal muscles. ${ }^{7}$ After passing through the pharyngeal musculature, it travels over the articulation of the temporal and sphenoid bones before penetrating the temporal bone and arriving at the middle ear. ${ }^{8}$
Somatic dysfunction of the temporal and sphenoid bones, as well as tension in the pharyngeal musculature, may cause impaired drainage of the eustachian tube as it passes through these structures. ${ }^{8}$ Eustachian tube dysfunction can also be caused by upper respiratory tract infections, altitude changes, allergies, a narrow eustachian tube, and large adenoids. ${ }^{8}$ In children, the eustachian tube is more vulnerable to decreased drainage because of its more horizontal positioning in the child's cranium. Osteopathic manipulative treatment may be an effective way to address biomechanical restrictions leading to impaired drainage, serous otitis, and otitis media.

This is a gentle technique that can be used in patients of all ages to assess and treat eustachian tube dysfunction. Contraindications may include mastoiditis, cellulitis, or open wounds in the areas where the physican's hands are placed. $^{3}$

Successful treatment may optimize eustachian tube function, improve inner ear drainage, and aid in more rapid symptomatic and physiologic improvement in patients with $\mathrm{AOM}^{3}$ The treatment performed in this video is not intended to treat any specific clinical condition and is only one aspect of the diagnosis and treatment plan an osteopathic physician may use to address the whole patient. (doi:10.7556/jaoa.2020.033)

\section{Acknowledgments}

We thank Jeff Reedy for video contributions. We also thank our child model and parent.

\section{References}

1. Hayes NM, Bezilla TA. Incidence of iatrogenesis associated with osteopathic manipulative treatment of pediatric patients. J Am Osteopath Assoc. 2006;106(10):605-608

2. Lund G, Carreiro JE. Characteristics of pediatric patients seen in medical school-based osteopathic manipulative medicine clinics. J Am Osteopath Assoc. 2010;110(7):376-380.

3. Seffinger MA, executive ed. Foundations of Osteopathic Medicine. 4th ed. Wolters Kluwer; 2017:1143. 
4. Steele KM, Carreiro JE, Viola JH, Conte JA, Ridpath LC. Effect of osteopathic manipulative treatment on middle ear effusion following acute otitis media in young children: a pilot study. J Am Osteopath Assoc. 2014;114(6):436-447. doi:10.7556/jaoa.2014.094

5. Mills MV, Henley CE, Barnes LL, Carreiro JE, Degenhardt BF. The use of osteopathic manipulative treatment as adjuvant therapy in children with recurrent acute otitis media. Arch Pediatr Adolesc Med. 2003;157 (9):861-866. doi:10.1001/archpedi.157.9.861

6. Degenhardt BF, Kuchera ML. Osteopathic evaluation and manipulative treatment in reducing the morbidity of otitis media: a pilot study. J Am Osteopath Assoc. 2006;106(6):327-334.
7. Leuwer R. Anatomy of the eustachian tube. Otolaryngol Clin North Am. 2016;49(5):1097-1106. doi:10.1016/j.otc.2016.05.002

8. Carreiro JE. An Osteopathic Approach to Children. Churchill Livingstone Elsevier; 2009:185-188

9. DiGiovanna EL., Schiowitz S, Dowling DJ, eds. An Osteopathic Approach to Diagnosis and Treatment. Lippincott Williams and Wilkins; 2005:615.

10. Nicholas AS, Nicholas EA, eds. Atlas of Osteopathic Techniques. Wolters Kluwer; 2016:511.

(c) 2020 American Osteopathic Association 